\title{
Study on the relationship between the workload and current implemented learning system of the students in the Dept. of Computer and Communication Systems Engineering, UPM
}

\begin{abstract}
Outcomes-based education (OBE) system is a motivated and reformed education system. OBE has been implemented in Bachelor of Engineering (B. Eng) programme offered by the Faculty of Engineering, Universiti Putra Malaysia since 2006. The learning process focuses on measuring the students' outcomes empirically. To contrast to OBE, the conventional learning system is primarily focuses on the resources available to students and to lecturers. In this paper, we present the relationship between the workload and the current implemented OBE learning system. Suggestions to improve the current OBE learning systems are also presented in order to enhance the learning method to benefit every entity involve in this system A survey was conducted to the student in the Department of Computer and Communication Systems Engineering. Most of the respondents do understand and aware about the concept of OBE since it was implemented. From the survey, more than $80 \%$ of the respondents aware the implementation of OBE in the department and $75 \%$ of them agree that OBE system has increased their workload.
\end{abstract}

Keyword: Maximum of five and separate with a semicolon 